\title{
Measurements of the Prompt Fission Neutron Spectrum at LANSCE: The Chi-Nu Experiment
}

\author{
Keegan J. Kelly ${ }^{1, \star}$, Matthew Devlin ${ }^{1}$, Jaime A. Gomez ${ }^{1}$, John M. O'Donnell ${ }^{1}$, Terry N. Taddeucci ${ }^{1}$, \\ Robert C. Haight ${ }^{1}$, Hye Young Lee ${ }^{1}$, Shea M. Mosby ${ }^{1}$, Nikolaos Fotiades ${ }^{1}$, Denise Neudecker ${ }^{1}$, \\ Patrick Talou ${ }^{1}$, Michael E. Rising ${ }^{1}$, Morgan C. White ${ }^{1}$, Clell J. Solomon ${ }^{1}$, Ching-Yen $\mathrm{Wu}^{2}$, Brian \\ Bucher $^{2}$, Matthew Q. Buckner ${ }^{2}$, and Roger A. Henderson ${ }^{2}$ \\ ${ }^{1}$ Los Alamos National Laboratory, Los Alamos, NM, 87544, USA \\ ${ }^{2}$ Lawrence Livermore National Laboratory, Livermore, CA, 94550, USA
}

\begin{abstract}
The goal of the Chi-Nu experiment at the Los Alamos Neutron Science Center is to measure the prompt fission neutron spectra from major actinides using a double time-of-flight method with a pulsed, white incoming neutron source. Fission events are detected with a parallel-plate avalanche counter and outgoing neutrons are detected with either a ${ }^{6} \mathrm{Li}$-glass or liquid scintillator detector array for low- or high-energy neutrons, respectively. A detector response matrix for the interaction of neutrons with the experimental environment for neutrons measured with the $\mathrm{Chi}-\mathrm{Nu}{ }^{6} \mathrm{Li}$-glass detector array has been calculated to obtain a full understanding of the measured Chi-Nu data and also to allow for nearly instantaneous production of simulated Chi-Nu data spectra. Prompt fission neutron spectra corresponding to 19 incoming neutron energy ranges from 0.7-20 $\mathrm{MeV}$ have been extracted using the ratio-of-ratios method with $\mathrm{Chi}-\mathrm{Nu}{ }^{6} \mathrm{Li}$-glass data on the neutron-induced fission of ${ }^{235} \mathrm{U}$.
\end{abstract}

\section{Introduction}

Accurate measurements of neutrons emitted promptly following neutron-induced fission of major actinides are required in order to understand the behavior of energetic nuclear systems and they are of interest for many other related applications. Furthermore, nuclear data evaluations are in need of new, high-precision measurements of prompt fission neutron spectra for ${ }^{239} \mathrm{Pu}$ and ${ }^{235} \mathrm{U}$ to differentiate between discrepant data sets and evaluations [1].

The Chi-Nu experiment is designed to measure the outgoing prompt fission neutron energy spectrum from $0.01-15 \mathrm{MeV}$ for an incoming neutron energy range from $0.7-20 \mathrm{MeV}$, effectively measuring the neutron $\chi$ matrix. The experimental setup for Chi-Nu will be described in Sect. 2. As is the case with most neutron-detection experiments, Chi-Nu uses neutron time of flight to measure the incoming and outgoing neutron energies. However, as will be discussed in Sect. 3, the scattering of neutrons off of objects in the experimental environment strongly distorts the measured experimental time-of-flight spectrum and results in time-of-flight energies that are lower than the desired, initial energy of the neutron as it was created. The approach taken during Chi-Nu data analysis to deal with

\footnotetext{
^e-mail: kkelly@lanl.gov
} 
this issue is to assemble a detector response matrix. The ratio-of-ratios method, a simple method for determining the underlying prompt fission neutron spectrum without assuming anything about the shape of said spectrum, is described in Sect. 4. Finally, results for the ${ }^{235} \mathrm{U}$ prompt fission neutron spectrum for outgoing neutron energies from 0.01-2.5 MeV are shown in Sect. 5.

\section{Experimental Description}

The Chi-Nu experiment is located on the $15 \mathrm{~L}$ beamline at the Weapons Neutron Research (WNR) Facility at the Los Alamos Neutron Science Center (LANSCE). This beamline receives a pulsed, white incoming neutron beam with a practical energy range of $\sim 100$ 's of $\mathrm{keV}-10$ 's of MeV. Incoming neutrons are created via proton-induced spallation reactions on a tungsten target and an incident neutron time-of-flight start time, referred to as $t_{0}$, is created via protons passing through an inductive coil prior to spallation during each pulse. These neutrons are then incident on a parallel-plate avalanche counter (PPAC) containing $10 \mathrm{Ti}$ foils (typically) electroplated with the actinide of interest [2]. Isobutane gas is continuously flowed through the PPAC, maintaining an approximate internal pressure of 4.2 Torr. A thin, grounded $\mathrm{Al}$ foil is placed on both sides of the Ti foil. A second Al foil is placed $3 \mathrm{~mm}$ away from each of the foils nearest to the target and a potential difference of - 375 Volts is applied between neighboring pairs of $\mathrm{Al}$ foils. A fission fragment traveling through the region between these $\mathrm{Al}$ foils ionizes the isobutane gas, creating an avalanche effect as the emitted electrons travel towards the grounded $\mathrm{Al}$ foil, thereby producing a fission signal and a fission signal time, $t_{f}$. The time difference $t_{f}-t_{0}$ yields the incoming neutron energy.

Prompt fission neutrons are emitted from the fission fragments on the order of $10^{-20}-10^{-18} \mathrm{~s}$ after creation of the fragments themselves. The neutrons are detected with one of two neutron-detector arrays. Low-energy neutrons $\left(E_{n}^{\text {out }} \leq 2.5 \mathrm{Mev}\right)$ are detected with a 22 -detector ${ }^{6} \mathrm{Li}$-glass array while high-energy neutrons $\left(E_{n}^{\text {out }} \geq 800 \mathrm{keV}\right)$ are detected with a 54-detector liquid scintillator array. This paper will focus on data acquired with the ${ }^{6} \mathrm{Li}$-glass detector array. The time associated with detection of the outgoing neutron is referred to as $t_{n}$ and the energy of the outgoing neutron is determined via the time difference $t_{n}-t_{f}[3,4]$. Backgrounds are measured with high-precision during data acquisition using a random coincidence method and subtracted from the measured data spectra. Details of this background measurement method can be found in Ref. [5] and will not be repeated here.

\section{The Chi-Nu ${ }^{6}$ Li-glass Detector Response Matrix}

The procedure for processing the experimentally-acquired detector signals to produce a backgroundsubtracted spectrum of neutron time-of-flight energies, $E_{t}$, is complicated and will not be explored here (see Ref. [6] for details). An example of a background-subtracted Chi-Nu ${ }^{6} \mathrm{Li}$-glass data spectrum corresponding to the prompt fission neutron spectrum of ${ }^{235} \mathrm{U}$ for an incoming neutron energy range of $1.0 \mathrm{MeV} \leq E_{n}^{i n c} \leq 1.5 \mathrm{MeV}$ is shown in Fig. 1. In ${ }^{6} \mathrm{Li}$-glass detectors, neutrons are detected via capture through the ${ }^{6} \mathrm{Li}(n, \alpha) t$ reaction, which has a large resonance at $\sim 240 \mathrm{keV}$ that is clearly visible in the measured spectrum. For reference, the convolution of the ENDF/B-VII.1 cross section for this reaction with a representative Maxwellian PFNS is shown in red in Fig. 1 overlayed on the data.

Following production of the measured $E_{t}$ spectrum, the data must be converted to the desired, initial neutron energy, $E$. It is implicitly assumed that $E=E_{t}$ when, for example, measurements are made relative to the time-of-flight spectrum obtained from a ${ }^{252} \mathrm{Cf}$ standard (see Eq. 1 of Ref. [7]). However, this assumption is incorrect for the majority of detected neutrons because it can be rare for a neutron to have exactly zero scatters between creation within the target and detection. In fact, for the $\mathrm{Chi}-\mathrm{Nu}{ }^{6} \mathrm{Li}$-glass array it has been shown that more than half of all detected neutrons have scattered at 


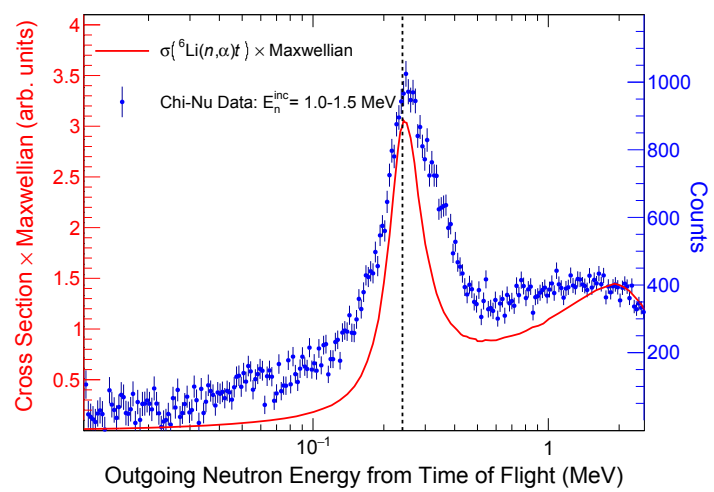

Figure 1. An example ${ }^{6} \mathrm{Li}$-glass spectrum as a function of $E_{t}$, the energy determined from the neutron time of flight. These data correspond to prompt fission neutrons emitted from the neutron-induced fission of ${ }^{235} \mathrm{U}$ for an incoming neutron energy range of $1.0 \mathrm{MeV} \leq E_{n}^{i n c} \leq 1.5 \mathrm{MeV}$. The data are shown as the blue circles. Additionally, the convolution of the ENDF/B-VII. $1{ }^{6} \mathrm{Li}(n, \alpha) t$ cross section with a PFNS known to approximately describe the displayed data is shown as the red, solid line. This line represents the expected counts from an "ideal" detector.

least once either off of an object in the experimental environment or off of material inside the detector itself before detection.

The effect of scattering can be observed from multiple features in the experimental time-of-flight spectrum shown in Fig. 1. The data are shown as the blue data points (described by the blue, righthand $y$ axis) and red line (described the red, left-hand y axis) shows the convolution of the ${ }^{6} \operatorname{Li}(n, \alpha) t$ cross section and a Maxwellian PFNS that is known to approximately describe the observed data. A similar figure to Fig. 1 has been shown in Ref. [8]. Note that the red line in Fig. 1 represents an "ideal" (zero scattering and perfect time resolution) detector. The most commonly-observed effect of scattering is an excess in counts at low energies (i.e. longer times of flight) compared to what would be expected from an ideal detector and this is clearly seen in Fig. 1. There are also possibly unexpected effects of scattering, such as an increase in counts at energies just above the ${ }^{6} \mathrm{Li}(n, \alpha) t$ resonance in Fig. 1. This may be counterintuitive because scattering acts to decrease the neutron energy. However, it can be shown that these counts are caused by neutrons that travel to a detector without scattering and with an energy just above the resonance energy. These neutrons may not immediately capture because they are off of the resonance, but they can quickly scatter off of oxygen, silicon, or other materials within the detector itself to reach the resonance energy, thereby capturing with a physical energy nearly equivalent to the resonance energy, but without significantly increasing the observed time of flight. In fact, all regions of the measured data spectrum are affected by this same neutron scattering effect, and neutrons with initial energy $E>2.5 \mathrm{MeV}$, the maximum plotted $E_{t}$, also appear in spectrum shown in Fig. 1.

Mathematically, this effect is best represented by the equation [7]

$$
C\left(p(E), E_{t}\right)=\int_{E t}^{\infty} p(E) \mathcal{R}\left(E, E_{t}\right) d E .
$$

Here, $C\left(p(E), E_{t}\right)$ is the number of observed counts in the measured $E_{t}$ data spectrum, which depends on the $E_{t}$ value being measured as well as the prompt fission neutron spectrum, $p(E)$, described as a function of the initial neutron energy, $E$. The function $\mathcal{R}\left(E, E_{t}\right)$ is the detector response matrix, which 
describes the probability for a neutron emitted with an energy $E$ to be detected with an energy $E_{t}$. The integration here is over the initial neutron energies and has a lower limit of $E_{t}$. This is because of the fact that a neutron detected with energy $E_{t}$ could not have been the result of neutron emitted with an energy $E<E_{t}$. However, it should be noted that experimental resolution effects blur this sharp energy boundary.

Typically, $\mathcal{R}\left(E, E_{t}\right)$ is only accessible through Monte Carlo simulations of the detector environment. The $\mathcal{R}\left(E, E_{t}\right)$ corresponding to the $\mathrm{MCNP}^{\circledR}$ simulation of the $\mathrm{Chi}-\mathrm{Nu}{ }^{6} \mathrm{Li}$-glass detector array is shown in Fig. 2. There are a number of features in this response matrix that are of note. First, the diagonal band of high counts corresponds to neutrons that were emitted from the target with energy $E$ and determined to have a time-of-flight energy $E_{t} \approx E$. Second, the streak of counts along $150 \mathrm{keV}$ $\leq E \leq 400 \mathrm{keV}$ corresponds the ${ }^{6} \mathrm{Li}(n, \alpha) t$ resonance at $\approx 240 \mathrm{keV}$. Finally, the fine structures along various $E$ values correspond either to resonances in materials in the experimental environment or to physical objects off of which neutrons are likely to scatter. For example, the thin structures at $E=35$ and $90 \mathrm{keV}$ correspond to resonances in ${ }^{27} \mathrm{Al}$ while, the thin structures at $E=2.5$ and $3.5 \mathrm{MeV}$ are believed to originate from scatters off of the outer can of the PPAC.

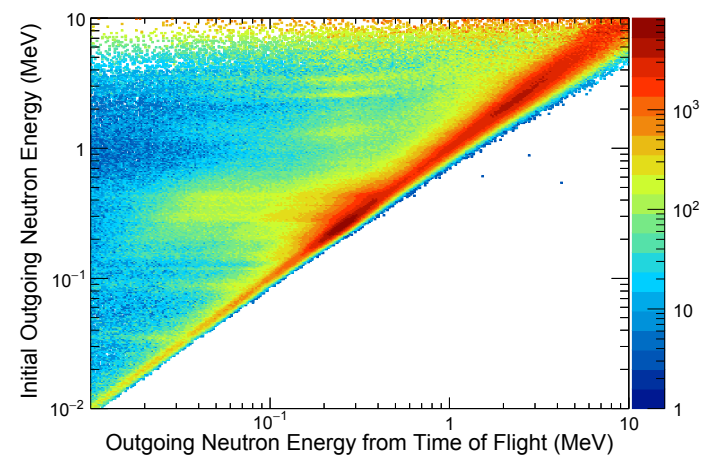

Figure 2. The neutron detector response function, $\mathcal{R}\left(E, E_{t}\right)$, for the Chi-Nu ${ }^{6} \mathrm{Li}$-glass array. See the text for a description of the main features.

In addition to providing useful information about the interaction of neutrons with the experimental environment, $\mathcal{R}\left(E, E_{t}\right)$ can also be used to rapidly produce simulated neutron spectra. Figure 3 shows a schematic description of how this process is carried out. First, the desired prompt fission neutron spectrum, $p(E)$, is chosen. The spectra shown in Fig. 3 correspond to Maxwellian distributions with various temperatures. Each row of the response matrix is then scaled by the value of $p(E)$ at that energy, $E$. Finally, each $E_{t}$ column is integrated over $E$ to acquire the total counts in each bin of the output simulated neutron spectrum. However, it is important to note that the uncertainty on the number of counts in each bin of these simulated spectra can be significantly more complicated than if an independent simulation was run. This procedure has been validated and described in further detail in Ref. [7].

\section{The Ratio-of-Ratios Method}

Although more sophisticated data-analysis procedures to obtain prompt fission neutron spectra from the acquired data spectra are under development at $\mathrm{Chi}-\mathrm{Nu}$, a surprisingly accurate result can be quickly obtained without assuming anything about the shape of the prompt fission neutron spectrum 


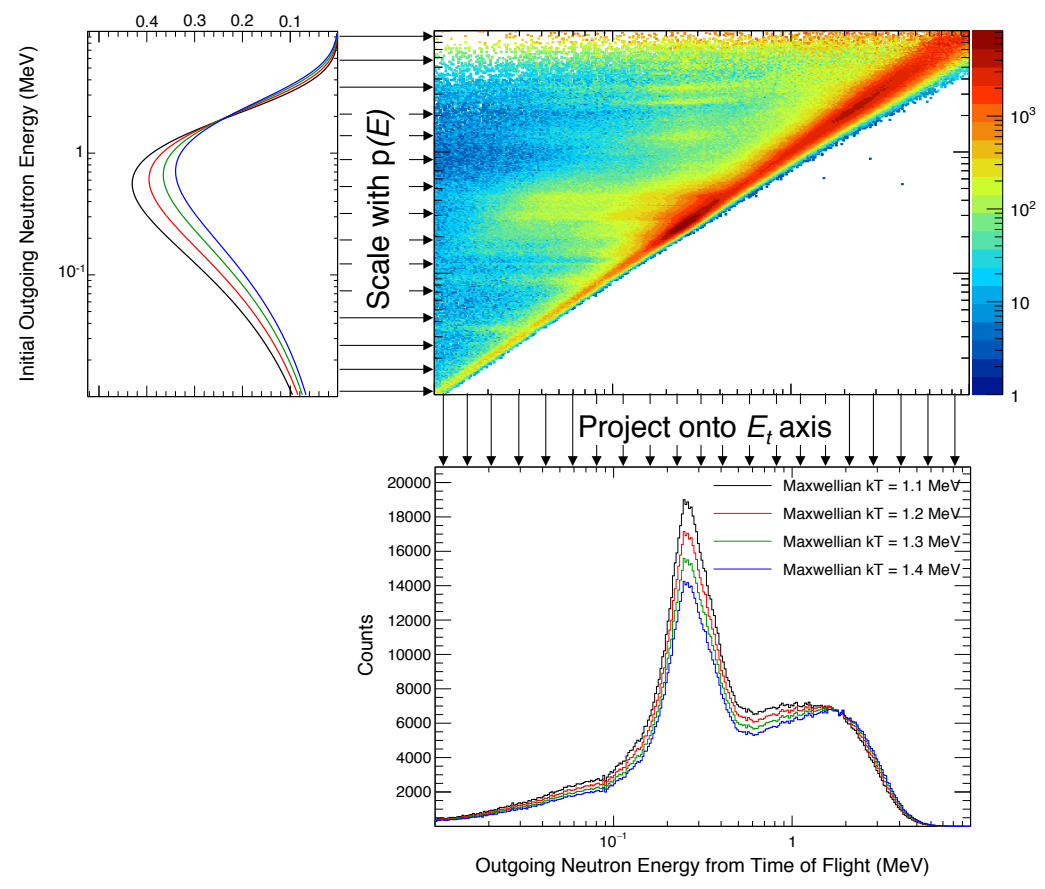

Figure 3. A schematic diagram of how $\mathcal{R}\left(E, E_{t}\right)$ can be used to rapidly produce simulated neutron spectra. See the text for a description.

using what is internally known as the "ratio-of-ratios method" [8]. This technique is based on the approximation that, for two different prompt fission neutron spectra, $p_{\alpha}(E)$ and $p_{\beta}(E)$, the ratio of the counts in each measured $E_{t}$ bin to the value of the appropriate prompt fission neutron spectrum evaluated at $E_{t}$ is equal for any $p_{\alpha}(E)$ and $p_{\beta}(E)$, i.e.

$$
\frac{C\left(p_{\alpha}(E), E_{t}\right)}{p_{\alpha}\left(E_{t}\right)} \approx \frac{C\left(p_{\beta}(E), E_{t}\right)}{p_{\beta}\left(E_{t}\right)} .
$$

Based on $\mathrm{MCNP}^{\circledR}$ simulations, this approximation is true within $\approx 5-10 \%$ below $E_{t}=200 \mathrm{keV}$ and is true to a higher precision above that energy for the Chi-Nu experimental environment with the ${ }^{6} \mathrm{Li}$-glass detectors using a range of possible prompt fission neutron spectrum shapes. If the same assumption is made for the experimental data, then it follows that

$$
\frac{C\left(p_{\alpha}(E), E_{t}\right)}{p_{\alpha}\left(E_{t}\right)} \approx \frac{C\left(p_{\exp }(E), E_{t}\right)}{p_{\text {exp }}\left(E_{t}\right)} \Rightarrow p_{\text {exp }}(E) \approx \frac{C\left(p_{\text {exp }}(E), E_{t}\right)}{C\left(p_{\alpha}(E), E_{t}\right)} p_{\alpha}\left(E_{t}\right) .
$$

In fact, it is Eq. 3 that is taken to be exactly true when prompt fission neutron spectra are measured relative to a ${ }^{252} \mathrm{Cf}$ standard. Rather than calculating the ratio shown in Eq. 2 for a single prompt fission neutron spectrum, such as ${ }^{252} \mathrm{Cf}$, this ratio is calculated for a set of prompt fission neutron spectra believed to span the range of possible prompt fission neutron spectrum shapes and averaged together in an attempt to more thoroughly assess the systematic uncertainty of this and similar methods given that the experimental prompt fission neutron spectrum cannot be assumed prior to analysis. This set 
of prompt fission neutron spectra are shown in Fig. 4. After averaging the ratio shown in Eq. 2 for the fission neutron spectra shown in Fig. 4, the expression for the experimental prompt fission neutron spectrum becomes

$$
p_{\text {exp }}(E)=C\left(p_{\text {exp }}(E), E_{t}\right)\left[\frac{1}{\kappa} \sum_{\alpha=1}^{\kappa} \frac{C\left(p_{\alpha}(E), E_{t}\right)}{p_{\alpha}\left(E_{t}\right)}\right]^{-1},
$$

where $\kappa$ is the total number of neutron spectra in Fig. 4 and the sum is over each spectrum, $\alpha$, shown in Fig. 4.

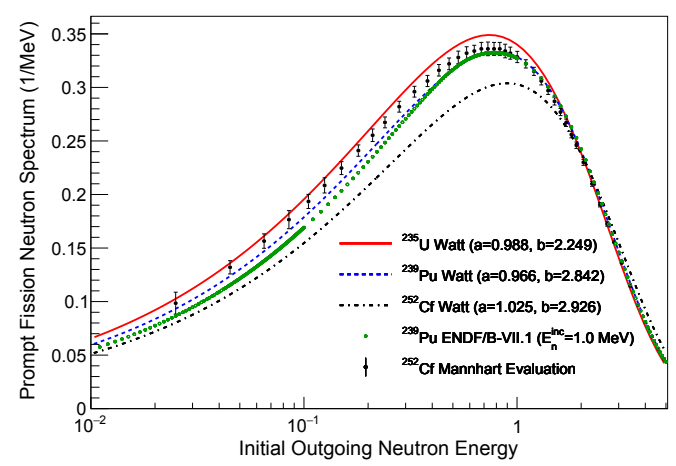

Figure 4. Shown here are the set of prompt fission neutron spectra considered for the ratio-of-ratios method. The Watt parameters, $a$ and $b[9,10]$, are in units of $\mathrm{MeV}^{-1}$. The ${ }^{239} \mathrm{Pu}$ ENDF/B-VII.1 evaluation spectrum is from Ref. [9] and the ${ }^{252} \mathrm{Cf}$ Mannhart evaluation can be found in Ref. [11].

\section{Low-Energy Results for ${ }^{235} \mathrm{U}$}

The method described in Sect. 4 was applied to ${ }^{6} \mathrm{Li}$-glass detector data collected from the neutroninduced fission of ${ }^{235} \mathrm{U}$. Prompt fission neutron spectra corresponding to 19 different incoming energy ranges from $0.7 \mathrm{MeV} \leq E_{n}^{i n c} \leq 20 \mathrm{MeV}$ were created. An example of one such prompt fission neutron spectrum corresponding to $2.0 \mathrm{MeV} \leq E_{n}^{i n c} \leq 3.0 \mathrm{MeV}$ is shown in Fig. 5 in black with the ENDF/BVII.1 [9] evaluations at $E_{n}^{i n c}=2.0$ and $3.0 \mathrm{MeV}$ shown as the solid, red line and the dashed, blue line, respectively. Reasonable agreement is obtained with the ENDF/B-VII.1 library for the shown prompt fission neutron spectrum with the exception of an excess observed in Chi-Nu data from 50 to $200 \mathrm{keV}$ and a slight decrease above $1 \mathrm{MeV}$. Additionally, a full covariance matrix has been produced for each of the 19 prompt fission neutron spectra measured for ${ }^{235} \mathrm{U}$. The covariance matrix corresponding to the spectrum shown in Fig. 5 is displayed in Fig. 6. Note that the increase in covariance at low energies is a result of a significant increase in background at those energies. Data from the Chi-Nu measurement of the ${ }^{235} \mathrm{U}$ prompt fission neutron spectrum for $E_{n}^{i n c}>5 \mathrm{MeV}$ will be included in the ENDF/B-VIII.0 evaluation (see Ref. [6]).

\section{Conclusions}

The Chi-Nu experiment at Los Alamos National Laboratory is designed to measure the prompt fission neutron spectra of major actinides with high accuracy and a detailed uncertainty quantification. In an 


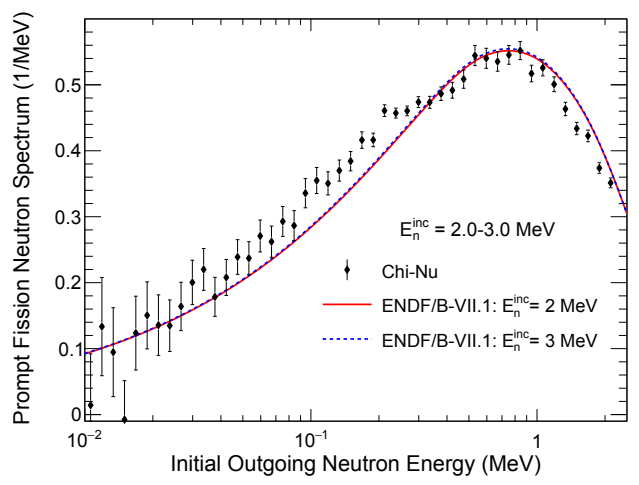

Figure 5. The prompt fission neutron spectrum of ${ }^{235} \mathrm{U}$ corresponding to an incoming neutron energy range of $2.0 \mathrm{MeV} \leq E_{n}^{\text {inc }} \leq 3.0 \mathrm{MeV}$ obtained using the $\mathrm{Chi}-\mathrm{Nu}{ }^{6} \mathrm{Li}$-glass detector array and the ratio-of-ratios method described in Sect. 4. The ENDF/B-VII.1 [9] evaluations corresponding to incoming neutron energies of 2.0 and 3.0 $\mathrm{MeV}$ are shown as the solid, red line and the dashed, blue line, respectively.

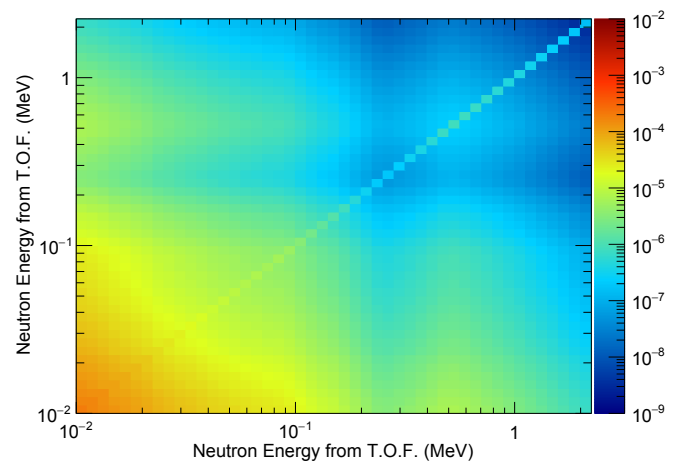

Figure 6. The covariance matrix corresponding to the prompt fission neutron spectrum shown in Fig. 5. Note that the increase in covariance at low energies is caused by a significant increase in the observed background at those energies.

effort to fully understand the interaction of neutrons measured at $\mathrm{Chi}-\mathrm{Nu}$ with the experimental environment, a detector response matrix has been constructed. This matrix also allows for rapid production of simulated neutron spectra from Chi-Nu detectors, which has proven to be useful for Chi-Nu data analysis. The ratio-of-ratios method has been applied to Chi-Nu ${ }^{6} \mathrm{Li}$-glass detector spectra collected on the neutron-induced fission of ${ }^{235} \mathrm{U}$. The results compare well to ENDF/B-VII.1 evaluation spectra and a large of portion of the available Chi-Nu data on ${ }^{235} \mathrm{U}$ will be included in the ENDF/B-VIII.0 evaluation. 


\section{Acknowledgements}

This work was performed under the auspices of the U.S. Department of Energy by Los Alamos National Laboratory under Contract DE-AC52-06NA25396 and by Lawrence Livermore National Security, LLC under contract DE-AC52-07NA27344.

\section{References}

[1] D. Neudecker, T.N. Taddeucci, R.C. Haight, H.Y. Lee, M.C. White et al., Nucl. Data Sheets 131, 289 (2016)

[2] C.Y. Wu, R.A. Henderson, R.C. Haight, H.Y. Lee, T.N. Taddeucci et al., Nucl. Instrum. and Methods A 794, 76 (2015)

[3] H.Y. Lee, T.N. Taddeucci, R.C. Haight, T.A. Bredeweg, A. Chyzh et al., Nucl. Instrum. and Methods A 703, 213 (2013)

[4] R.C. Haight, C.Y. Wu, H.Y. Lee, T.N. Taddeucci, B.A. Perdue et al., Nucl. Data Sheets 123, 130 (2015)

[5] J. O'Donnell, Nucl. Instrum. and Methods A 805, 87 (2016)

[6] M. Devlin, J.A. Gomez, K.J. Kelly, R.C. Haight, J.M. O’Donnell et al., Nucl. Data Sheets (to appear)

[7] K.J. Kelly, J.M. O’Donnell, J.A. Gomez, T.N. Taddeucci, M. Devlin et al., Nucl. Instrum. and Methods A 866, 182 (2017)

[8] T.N. Taddeucci, R.C. Haight, H.Y. Lee, D. Neudecker, J.M. O’Donnell et al., Nucl. Data Sheets 1232, 135 (2015)

[9] M.B. Chadwick, M. Herman, P. Oblŏzinský, M.E. Dunn, Y. Danon et al., Nucl. Data Sheets 112, 2887 (2011)

[10] B.E. Watt, Phys. Rev. 87, 1037 (1952)

[11] W. Mannhart, Properties of Neutron Sources, IAEA-TECDOC-410 pp. 158-171 (1987) 